\title{
Transverse tensile behaviour of unidirectional plies reinforced with flax fibres
}

\author{
Christophe Baley $^{a},{ }^{*}$, Yves Perrot $^{a}$, Frederic Busnel $^{a}$, Herve Guezenoc ${ }^{a}$ and Peter Davies ${ }^{b}$
}

\author{
a Université de Bretagne Sud, L2PIC, BP 92116, 56321, Lorient Cedex, France \\ ${ }^{\mathrm{b}}$ IFREMER, Materials and Structures group (ERT/MS), BP70, 29280 Plouzané, France
}

*: Corresponding author : Christophe Baley, Tel.: +33 2978745 53; fax: +33 2978745 88, email address : christophe.baley@univ-ubs.fr

\begin{abstract}
:
Flax provides a renewable source of fibres with good mechanical properties, which justifies its use as a reinforcement for polymers. Composite materials made with flax which has only undergone mechanical treatments (stripping followed by combing) results in the presence of bundles of fibres. When a unidirectional flax/polyester ply is loaded in transverse tension the damage mechanisms are complex; cracks develop in the matrix, and at the fibre/matrix interface, but they also appear within the fibres themselves and in the lamellae within the fibre bundles. This emphasises the highly anisotropic nature of these flax fibres, whose average longitudinal Young's modulus is $59 \mathrm{GPa}$ and whose transverse modulus is estimated here to be $8 \mathrm{GPa}$.
\end{abstract}

Keywords: Composite materials; Flax; Glass; Polyester; Transverse tensile; Unidirectional 


\section{Introduction}

Flax fibres show many advantages as a reinforcement for polymers, including good mechanical properties [1], low density $\left(1500 \mathrm{~kg} / \mathrm{m}^{3}\right)$ and biodegradability. They require little energy to produce and come from a renewable source. The structure of flax fibres is complex and similar to that of a composite material reinforced by helical cellulose fibres held in place by a matrix of polymers (hemicellulose, lignin, pectines). They may therefore be considered as natural biocomposites (nano biocomposites). As a result flax fibres are highly anisotropic as will be shown below.

In the plant, short fibres (33 $\mathrm{mm}$ on average) of polygonal section are assembled in bundles (Fig. 1). When composites are produced using fibres which have only been treated mechanically (stripping of the fibrous elements from the plant stem followed by combing) these bundles are found in the composite, (Fig. 2). Within a bundle the fibres are bonded together by lamellae.

The properties of composite materials reinforced by unidirectional (UD) fibres are known to be highly anisotropic with high values of stiffness and strength in the fibre direction and poor mechanical behaviour in the transverse direction. Low transverse tensile strength is a major weakness of composites. The presence of fibres results in stress concentrations and the strength is lower than that of the unreinforced matrix. For a multi-directional composite with plies in different directions the first damage (first ply failure) often corresponds to the transverse tensile strength of the plies with unidirectional reinforcement [2].

In the present letter the transverse tensile behaviour of a UD ply reinforced with flax fibres is considered, in order to characterize the behaviour of these materials for structural applications. The influence of fibre bundles and anisotropy have received particular attention. First, macroscopic flax reinforced composite transverse properties are presented. Then by using micromechanics expressions the transverse tensile modulus of the fibres has been estimated. Finally transverse damage mechanisms are observed and a particular type of damage is noted. For comparison, tests have also been performed on UD glass/polyester materials.

\section{Experimental procedure}

\subsection{Materials and preparation}

The flax fibres were taken from flax plants of the Ariane variety grown in Normandy (France). The fibres were subjected to dew retting, then the fibrous elements were stripped from the plant and combed. The E-glass fibres were coated with a textile-plastic size compatible with polyester matrix resins (Saertex - Unidirectional-Glass-Fabric). Layers of fibres with area weight of $540 \mathrm{~g} / \mathrm{m}^{2}$ were stitched together by glass $\left(50 \mathrm{~g} / \mathrm{m}^{2}\right)$, there are no transverse fibres. This type of reinforcement allowed the same specimen manufacturing route to be used for glass and flax fibres. The resin was an orthophthalic polyester (Polylite 420 M 731).

The composite panels were produced in a press with spacers to define thickness and hence fibre content. Fibre content was measured by burn-off tests on 5 samples. Unidirectional composites, reinforced with glass fibres, consisted of 5 reinforcement layers. After cure at room temperature samples were post-cured for 12 hours at $65^{\circ} \mathrm{C}$. 


\subsection{Test methods}

Tensile tests on composites were performed according to French standard NF T 57-101 on parallel sided specimens of width $25 \mathrm{~mm}$. Elongation was measured using an extensometer. Loading rate was $1 \mathrm{~mm} /$ minute.

In order to observe the damage mechanisms samples were also tested on a small tensile test machine (DEBEN microtest) inside a scanning electron microscope (SEM), a Jeol JSM $6460 L$.

\section{Results and discussion}

\subsection{Transverse tensile properties of a UD ply}

Fig. 3 shows the stress-strain behaviour of UD plies tested in the transverse direction. Table 1 summarises the results obtained.

For the same fibre volume content $(21.5 \%)$, these results show :

A higher stiffness and strength for the glass reinforced composites (Table 1)

A higher failure strain for the flax reinforced composite (Table 1)

A nearly linear stress-strain behaviour for the glass reinforced composite but non-linear behaviour for the flax reinforced materials (Fig. 3).

These values are similar to those usually measured on glass reinforced UD composites, for which transverse failure strains of $0.3 \%$ have been reported for a polyester matrix (with $30 \%$ fibre reinforcement by weight) [3].

Many previous studies have examined the parameters which influence transverse tensile strength such as :

Matrix failure strain [4],

The quality of the fibre/matrix interface [4] and that of an interphase [5] [6],

Fibre content [7],

Fibre distribution in the composite, fibre and resin zones rich regions are often noted [8],

Fibre diameter variation [9],

Loading temperature [7],

Residual stresses [5] which have two sources: resin volume shrinkage and the difference between manufacturing and testing temperatures.

The presence of microcracks, Sjögren [10] revealed the presence of defects (fibre-matrix debonds, matrix cracks) in a glass/polyester laminate just after cure.

- The presence of porosity [8]. Defects modify the mechanical properties. The origins of these defects depend on the material components, the cure cycle and the impregnation method [11].

The heterogeneity of composite materials is the main cause of failure of UD plies loaded in transverse tension. Stress and strain concentrations are induced in the matrix between the fibres. De Kok has shown that a global ply strain of $1 \%$ can result in local strains greater than $5 \%$ [12]. An irregular fibre distribution accentuates this effect. As a result of these strain concentrations the transverse failure strain for UD plies is considerably lower than the longitudinal failure strain.

In a glass fibre reinforced composite the first microcracks to appear are generally located at or near the fibre/matrix interface (often described as debonding). The initiation of microcracks is therefore controlled by the adhesion between fibre and matrix and by the quality of the interface or interphase. These first cracks are often seen in transition zones between fibre- 
rich and resin-rich areas [13]. This phenomenon accentuates the influence of local heterogeneity. Residual stresses may partly explain this effect [14]. During manufacture the matrix contracts more than the fibres (shrinkage of the resin and higher thermal expansion coefficient than the fibres). This contraction introduces compression in the direction radial to the fibres. However, in the transition zone where debonding initiates, the matrix contraction is higher than in the fibre rich zones so tensile residual stresses develop [13].

The fibre content $\left(\mathrm{V}_{\mathrm{f}}\right)$ has a strong influence on the transverse failure strain of UD plies, the strain decreasing as fibre content increases (Fig. 4). Kies proposed an analytical model to determine the strain concentration factor [15]. However, this is not applicable here as certain assumptions such as a uniform fibre distribution, absence of porosity and no residual stresses are not respected. Certain flax fibres also have a central lumen which may be considered as a void.

While the longitudinal modulus of flax fibres can be measured directly, the transverse modulus is more difficult to determine. However, it can be estimated from micro-mechanics equations (Halpin \& Tsai, Hopkins \& Chamis) using the macroscopic response obtained during a transverse tensile test on a UD ply. These equations assume ideal composites, thus Halpin and Tsai [16] consider the fibres to be of constant diameter and regularly distributed in the matrix with a triangular or square motif. These are clearly simplifying assumptions and the values obtained from the micro-mechanics approach must be treated as estimations only. Nevertheless they provide ballpark figures for these properties.

The equation proposed by Halpin and Tsai is the following :

$$
\frac{M}{M_{m}}=\frac{1+\xi * \eta * V_{f}}{1-\eta^{*} V_{f}}
$$

where,

$$
\eta=\frac{\frac{M_{f}}{M_{m}}-1}{\frac{M_{f}}{M_{m}}+\xi}
$$

and

$$
\begin{aligned}
& M=E_{L}, E_{T} \text { or } G_{L T} \\
& M_{f}=E_{f} \text { or } G_{f} \text { respectively } \\
& M_{m}=E_{m} \text { or } G_{m} \text { respectively } \\
& m, f, L, T \text { correspond to matrix, fibre, longitudinal and transverse directions. } \\
& V_{f}=\text { fibre volume content } \\
& \xi=\text { shape factor }
\end{aligned}
$$

Another expression, proposed by Hopkins and Chamis [17] has also been applied here. The composite transverse Young's modulus, $\mathrm{E}_{\mathrm{T}}$, can then be expressed as : 
$E_{T}=E_{m}\left[\left(1-\sqrt{V_{f}}\right)+\frac{\sqrt{V_{f}}}{\left(1-\sqrt{V_{f}\left(1-\frac{E_{m}}{E_{f T}}\right)}\right)}\right]$

$E_{f T}$ is calculated using $E_{m}$ (3152 Mpa here), $V_{f}$ and $E_{T}$ as input data.

The transverse modulus of flax fibres estimated using the Halpin-Tsai expression is $7 \mathrm{GPa} \pm$ 2, and using the Hopkins-Chamis expression $9.5 \mathrm{GPa} \pm 4$. These estimations are similar to the value for jute fibres estimated by Cichosky to be $5.5 \mathrm{GPa}$ [18]. The longitudinal Young's modulus measured on these flax fibres is $59 \mathrm{GPa}$ [1], so they are clearly highly anisotropic.

\subsection{Damage mechanisms}

When tensile tests are performed in the SEM it is observed that crack propagation in a UD flax/polyester ply (Fig. 5) crosses fibre bundles (point A), runs along the fibre-matrix interface (point $B$ ), but also runs through single fibres (point $C$ ) as well as propagating in the matrix. Detailed examination of the damage development within a fibre bundle also reveals that the fibres delaminate. The presence of the lumen at the centre of these fibres appears to encourage crack initiation. (Fig. 6). This can be explained by the multilayer structure of these fibres but introduces a new damage mechanism not seen in conventional composites.

In order to prevent cracking under transverse tensile loads within structural components it is necessary to define criteria which account for the response of the different components. This is possible in conventional composites though the radial tensile strength of the interphase is particularly difficult to characterize. For natural fibres it is important to account for the properties of the lamellae and of the fibres themselves. Given the multilayer nature of flax fibres (composites with primary and secondary layers, the secondary layer consisting of 3 layers), the transverse strength of the fibre will be difficult to measure and this area requires further study. It should also be noted that the water absorbed by these fibres strongly affects their strength [19], and cannot be ignored.

\section{Conclusions}

With a polyester matrix UD composites reinforced by flax fibres show a higher failure strain than glass reinforced composites with the same fibre content. This is a useful result as this property is related to the damage threshold of multidirectional composites.

The composites studied here were reinforced by bundles of fibres, as only mechanically stripped and combed flax was used. The fibre structure can be considered as a multilayer composite reinforced by cellulose fibrils. These fibres are assembled by lamellae and made up of primary and secondary layers, the latter being composed of three other layers. Flax fibres are highly anisotropic, their mean longitudinal Young's modulus is $59 \mathrm{GPa}$, the transverse modulus is estimated to be $8 \mathrm{GPa}$.

Damage under transverse loading is observed as traditionally observed matrix and interface cracks, but also cracks within fibre bundles and in the fibres themselves. The latter is important as it suggests a limit to the improvement which can be made in transverse tensile properties. 
In order to optimise the properties of flax reinforced composites improved treatments are needed to separate fibres, clean their surfaces, and reduce their hydrophilic tendency. The reinforcement by individual fibres, rather than bundles, should improve dispersion and eliminate one of the damage mechanisms observed here, the crack propagation through the bundles. However, particular attention must now be paid to the fibre delamination mechanism if satisfactory composite performance is to be achieved.

\section{References}

1. C. Baley, Composites Part A 33 (2002) 939-948.

2. R.F. Gibson, McGraw-Hill International Editions (New York, 1994).

3. R.M. Mayer, Bourne Press Ltd. The Design Council (London, 1993).

4. K. Benzarti, L. Cangemi, F. Dal Maso, Composites Part A 32 (2001) 197-206.

5. J.M.M. de Kok, H.E.H. Meijer, Composites Part A 30 (1999) 917-932.

6. S. Keusch, R. Haessler, Composites Part A 30 (1999) 997-1002.

7. J.M.M. de Kok, H.E.H. Meijer, Composites Part A 30 (1999) 905-916.

8. L. Zhang, L.J. Ernst, H.R. Brouwer, Mechanics of Materials 27 (1998) 13-26.

9. A.A. Gusen, P.J. Hine, I.M. Ward, Composite Science and Technology 60 (2000) 535-541.

10. B.A. Sjögren, L.A. Berglund, Composites Science and Technology 60 (2000) 9-21.

11. C. Baley, P. Davies, Y. Grohens, G. Dolto, Applied Composite Materials 11 (2004) 96126.

12. J.M.M. de Kok, Ph.D. Thesis, Eindhoven University of Technology, The Nederlands (1995).

13. P. Meurs, Ph.D. Thesis, Eindhoven University of Technology, The Nederlands (1998).

14. W.L. Bradley, C. Wood, C. Chatawanich, Proceeding of ICCM-10, Whistler BC, Canada, 311-318.

15. J.A. Kies, US Naval Research Laboratory Report Nº 5752 (1962).

16. J.C. Halpin, J.L. Kardos, Polym. Eng. Sci. 16 (5) (1976) 344-352.

17. D.A. Hopkins, C.C. Chamis, ASTM Philadelphia, STP 964 (1998) 159-176.

18. F.R. Cichosky, J.L. Thomason, Composite Science and Technology 62 (2002) 669-678.

19. C. Baley, C. Morvan, Y. Grohens, Macromolecular Symposia 222 (2005) 195-201. 


\section{Figures}

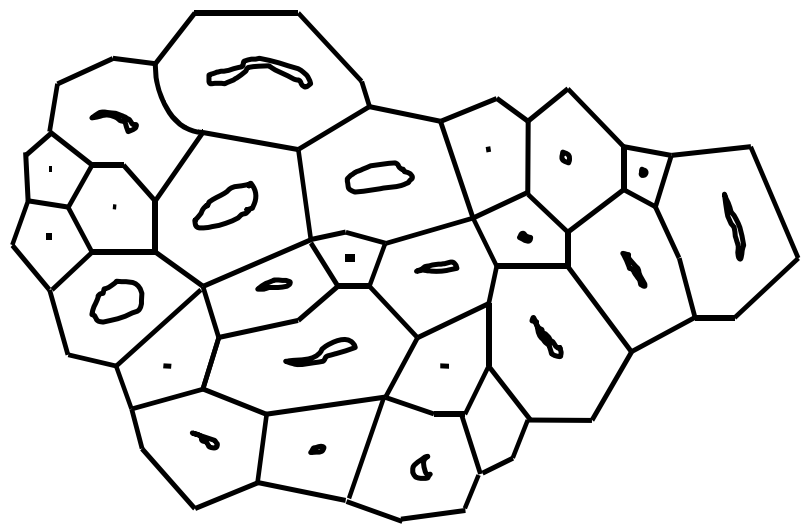

Fig. 1. Section of a flax fibre bundle

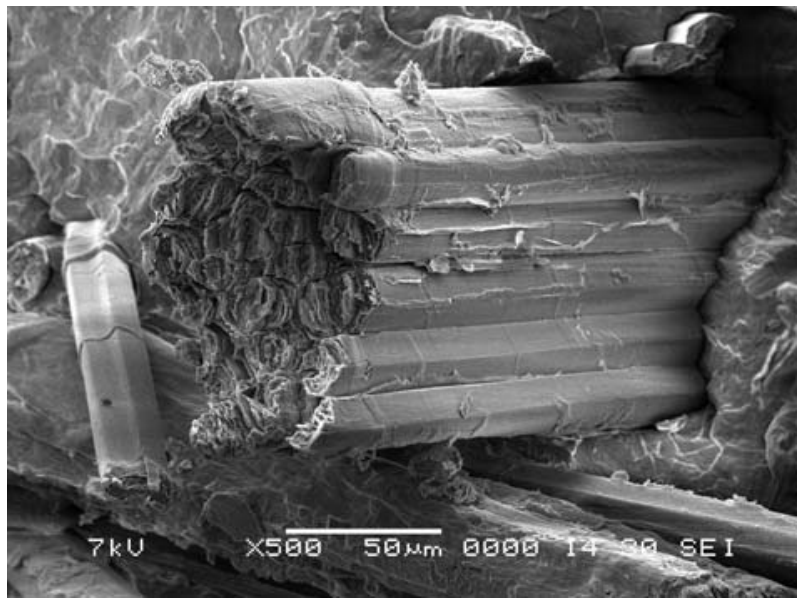

Fig. 2. Example of flax fibre bundle in a composite.

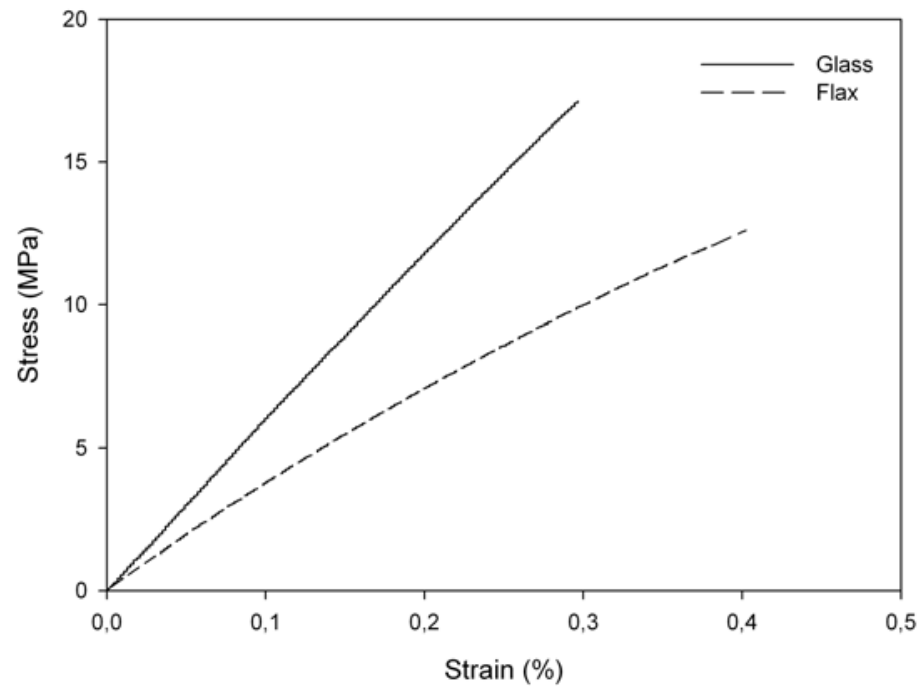

Fig. 3. Transverse stress-strain plots for glass and flax UD plies, Fibre volume fraction 0.21. 


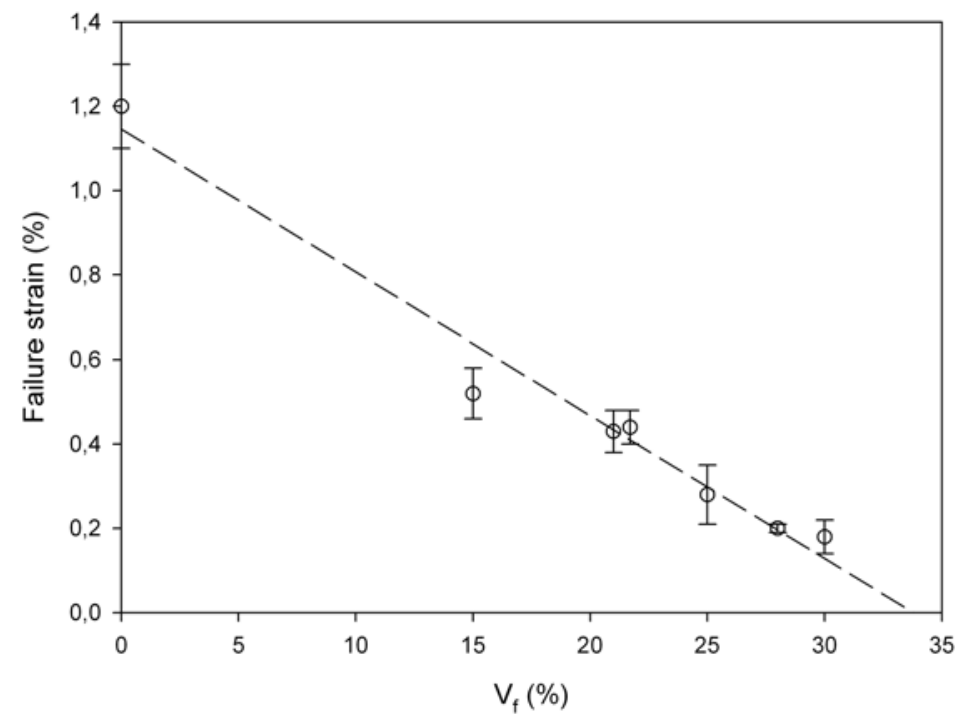

Fig. 4. Unidirectional flax/polyester loaded in transverse tension. Influence of fibre volume fraction on failure strain. Line indicates best fit.

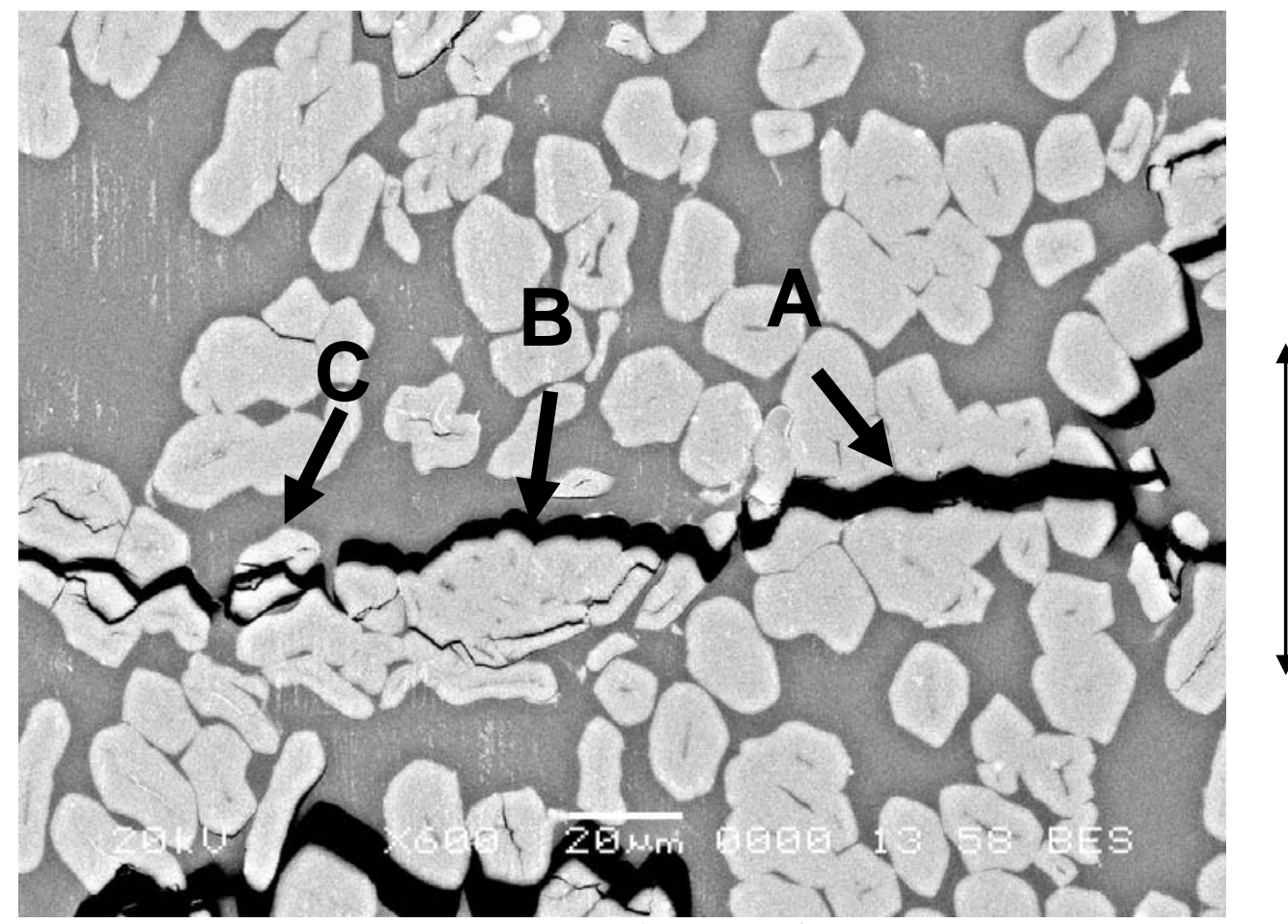

Loading direction

Fig. 5. Transverse tensile test in the SEM, flax/polyester. Development of crack in a UD ply within a bundle of flax fibres (A), at the fibre-matrix interface (B), and failure within a single fibre (C). 


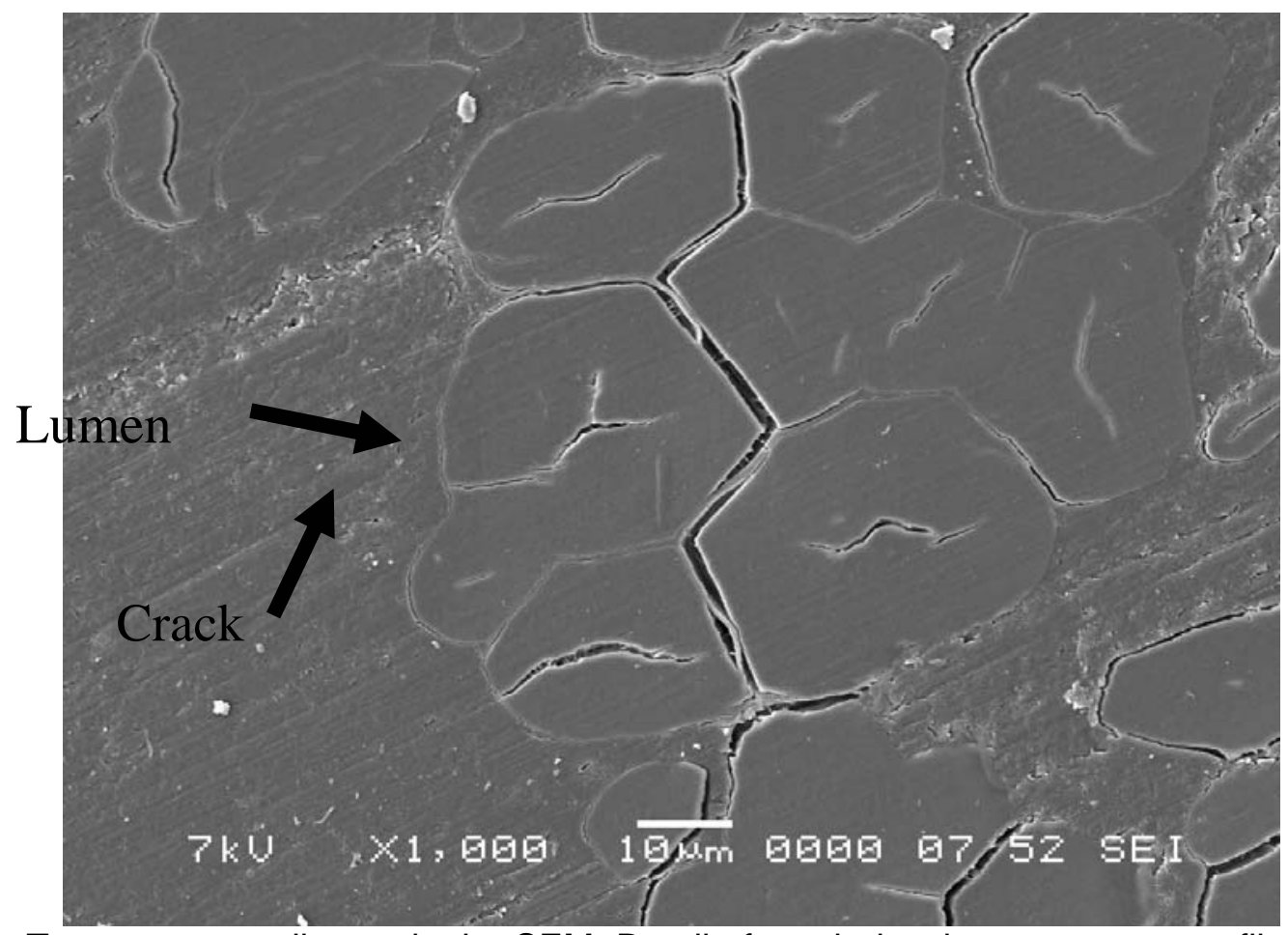

Fig. 6. Transverse tensile test in the SEM. Detail of crack development across a fibre bundle and cracks in individual fibres.

\section{Tables}

\begin{tabular}{ccccc}
\hline Description & $\mathrm{V}_{\mathrm{f}}(\%)$ & $\mathrm{E}(\mathrm{MPa})$ & Failure stress (MPa) & Failure strain (\%) \\
\hline Polyester/flax & 21.7 & $3622 \pm 280$ & $12.6 \pm 0.6$ & $0.44 \pm 0.04$ \\
\hline Polyester/glass & 21.4 & $5982 \pm 375$ & $15.2 \pm 2.5$ & $0.26 \pm 0.04$ \\
\hline
\end{tabular}

Table 1. Comparison of mean transverse tensile properties of UD plies reinforced with glass or flax fibres for the same fibre volume fraction. ( \pm standard deviation). 